\title{
Two-photon physics
}

\author{
M. Vanderhaeghen ${ }^{\mathrm{a}}$ \\ 12000 Jefferson Ave, Newport News, VA 23606, USA \\ / \\ Published online: 15 May 2006 - (c) Società Italiana di Fisica / Springer-Verlag 2006
}

Physics Department, The College of William \& Mary, Williamsburg, VA 23187, USA and Theory Group, Jefferson Lab,

\begin{abstract}
It is reviewed how Compton scattering sum rules relate low-energy nucleon structure quantities to the nucleon excitation spectrum. In particular, the GDH sum rule and recently proposed extensions of it will be discussed. These extensions are sometimes more calculationally robust, which may be an advantage when estimating the chiral extrapolations of lattice QCD results, such as for anomalous magnetic moments. Subsequently, new developments in our description of the nucleon excitation spectrum will be discussed, in particular a recently developed chiral effective field theory framework for the $\Delta(1232)$-resonance region. Within this framework, we discuss results on $N$ and $\Delta$ masses, the $\gamma N \Delta$ transition and the $\Delta$ magnetic dipole moment.
\end{abstract}

PACS. 25.20.Dc Photon absorption and scattering - 12.39.Fe Chiral Lagrangians - 13.40.Gp Electromagnetic form factors - 13.40.Em Electric and magnetic moments

\section{Introduction}

Sum rules for Compton scattering off a nucleon offer a unique tool to relate low energy nucleon structure quantities to the nucleon excitation spectrum [1]. E.g., the Gerasimov, Drell, Hearn (GDH) sum rule (SR) [2] relates a system's anomalous magnetic moment to a weighted integral over a combination of doubly polarized photoabsorption cross sections. Impressive experimental programs to measure these photoabsorption cross-sections for the nucleon have recently been carried out at ELSA and MAMI (for a review see ref. [3]). Such measurements provide an empirical test of the GDH SR, and can be used to generate phenomenological estimates of electromagnetic polarizabilities via related SRs. The GDH SR is particularly interesting because both its left- and right-hand-sides can be reliably determined, thus providing a useful verification of the fundamental principles (such as unitarity and analyticity) which go into its derivation. At the present time, it has been established that the proton sum rule is satisfied within the experimental precision, while the case is still out for the neutron.

After a lightning review of the GDH and related sum rules in sect. 2, I discuss a recently proposed linearized version of the GDH sum rule $[4,5]$. When applying this new sum rule to the nucleon in the context of chiral perturbation theory, it allows for an elementary calculation (to one loop) of quantities such as magnetic moments and polarizabilities to all orders in the heavy-baryon expansion. The

\footnotetext{
a e-mail: marcvdh@jlab. org
}

chiral behavior of the nucleon magnetic moment allows to make a link with lattice QCD calculations.

Subsequently, the nucleon excitation spectrum is discussed in sect. 3. Many Compton scattering sum rules, such as the GDH sum rule, are dominated by the $\Delta(1232)$ resonance. I discuss a recently proposed relativistic chiral effective field theory as a new systematic framework to both extract resonance properties from the experiment and to perform a chiral extrapolation of lattice QCD results for those resonance properties.

\section{Sum rules in Compton scattering}

\subsection{Derivation of forward Compton scattering sum rules}

The forward-scattering amplitude describing the elastic scattering of a photon on a target with spin $s$ (real Compton scattering) is characterized by $2 s+1$ scalar functions which depend on a single kinematic variable, e.g., the photon energy $\nu$. In the low-energy limit each of these functions corresponds to an electromagnetic moment - charge, magnetic dipole, electric quadrupole, etc. - of the target. In the case of a spin- $1 / 2$ target, such as the nucleon, the forward Compton amplitude is generally written as

$$
T(\nu)=\varepsilon^{\prime *} \cdot \varepsilon f(\nu)+i \boldsymbol{\sigma} \cdot\left(\varepsilon^{\prime *} \times \varepsilon\right) g(\nu),
$$

where $\varepsilon, \varepsilon^{\prime}$ is the polarization vector of the incident and scattered photon, respectively, while $\boldsymbol{\sigma}$ are the Pauli matrices representing the dependence on the target spin. The 
crossing symmetry of the Compton amplitude of eq. (1) means invariance under $\varepsilon^{\prime} \leftrightarrow \varepsilon, \nu \leftrightarrow-\nu$, which obviously leads to $f(\nu)$ being an even and $g(\nu)$ being an odd function of the energy : $f(\nu)=f(-\nu), g(\nu)=-g(-\nu)$. The two scalar functions $f(\nu), g(\nu)$ admit the following low-energy expansion:

$$
\begin{aligned}
& f(\nu)=-\frac{e^{2}}{4 \pi M}+\left(\alpha_{E}+\beta_{M}\right) \nu^{2}+\mathcal{O}\left(\nu^{4}\right), \\
& g(\nu)=-\frac{e^{2} \kappa^{2}}{8 \pi M^{2}} \nu+\gamma_{0} \nu^{3}+\mathcal{O}\left(\nu^{5}\right),
\end{aligned}
$$

and hence, in the low-energy limit, are given in terms of the target's charge $e$, mass $M$, and anomalous magnetic moment (a.m.m.) $\kappa$. The next-to-leading order terms are given in terms of the nucleon electric $\left(\alpha_{E}\right)$, magnetic $\left(\beta_{M}\right)$, and forward spin $\left(\gamma_{0}\right)$ polarizabilities.

In order to derive sum rules (SRs) for these quantities one assumes the scattering amplitude is an analytic function of $\nu$ everywhere but the real axis, which allows writing the real parts of the functions $f(\nu)$ and $g(\nu)$ as a dispersion integral involving their corresponding imaginary parts. The latter, on the other hand, can be related to combinations of doubly polarized photoabsorption crosssections via the optical theorem,

$$
\begin{aligned}
& \operatorname{Im} f(\nu)=\frac{\nu}{8 \pi}\left[\sigma_{1 / 2}(\nu)+\sigma_{3 / 2}(\nu)\right], \\
& \operatorname{Im} g(\nu)=\frac{\nu}{8 \pi}\left[\sigma_{1 / 2}(\nu)-\sigma_{3 / 2}(\nu)\right],
\end{aligned}
$$

where $\sigma_{\lambda}$ is the doubly-polarized total cross-section of the photoabsorption processes, with $\lambda$ specifying the total helicity of the initial system. Averaging over the polarization of initial particles gives the total unpolarized cross-section, $\sigma_{T}=\frac{1}{2}\left(\sigma_{1 / 2}+\sigma_{3 / 2}\right)$.

After these steps one arrives at the results (see, e.g., [1] for more details):

$$
\begin{aligned}
& f(\nu)=f(0)+\frac{\nu^{2}}{2 \pi^{2}} \int_{0}^{\infty} \frac{\sigma_{T}\left(\nu^{\prime}\right)}{\nu^{2}-\nu^{2}-i \epsilon} \mathrm{d} \nu^{\prime}, \\
& g(\nu)=-\frac{\nu}{4 \pi^{2}} \int_{0}^{\infty} \frac{\Delta \sigma\left(\nu^{\prime}\right)}{\nu^{\prime 2}-\nu^{2}-i \epsilon} \nu^{\prime} \mathrm{d} \nu^{\prime}
\end{aligned}
$$

with $\Delta \sigma \equiv \sigma_{3 / 2}-\sigma_{1 / 2}$, and where the sum rule for the unpolarized forward amplitude $f(\nu)$ has been oncesubtracted to guarantee convergence. These relations can then be expanded in energy to obtain the SRs for the different static properties introduced in eqs. (2), (3). In this way we obtain the Baldin SR $[6,7]$ :

$$
\alpha_{E}+\beta_{M}=\frac{1}{2 \pi^{2}} \int_{0}^{\infty} \frac{\sigma_{T}(\nu)}{\nu^{2}} \mathrm{~d} \nu,
$$

the GDH SR:

$$
\frac{e^{2} \kappa^{2}}{2 M^{2}}=\frac{1}{\pi} \int_{0}^{\infty} \frac{\Delta \sigma(\nu)}{\nu} \mathrm{d} \nu,
$$

a SR for the forward spin polarizability:

$$
\gamma_{0}=-\frac{1}{4 \pi^{2}} \int_{0}^{\infty} \frac{\Delta \sigma(\nu)}{\nu^{3}} \mathrm{~d} \nu,
$$

and, in principle, one could continue in order to isolate higher-order moments [8].

Recently, the helicity difference $\Delta \sigma$ which enters the integrands of eqs. (9) and (10) has been measured. The first measurement was carried out at MAMI (Mainz) for photon energies in the range $200 \mathrm{MeV}<\nu<800 \mathrm{MeV}[9$, 10], and was extended at ELSA (Bonn) [11] for $\nu$ up to $3 \mathrm{GeV}$. This difference, shown in fig. 1, fluctuates much more strongly than the total cross section $\sigma_{T}$. The threshold region is dominated by $S$-wave pion production, and therefore mostly contributes to the cross section $\sigma_{1 / 2}$. In the region of the $\Delta(1232)$ with spin $J=3 / 2$, both helicity cross sections contribute, but since the transition is essentially $M 1$, we find $\sigma_{3 / 2} / \sigma_{1 / 2} \approx 3$. As seen from fig. $1, \sigma_{3 / 2}$ also dominates the proton photoabsorption cross section in the second and third resonance regions.

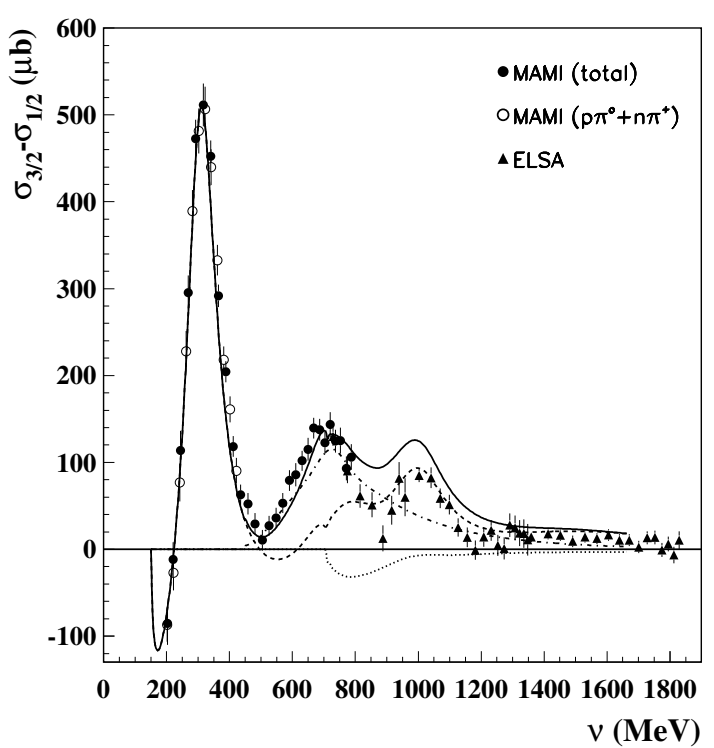

Fig. 1. The helicity difference $\sigma_{3 / 2}(\nu)-\sigma_{1 / 2}(\nu)$ for the proton. The calculations include the contribution of $\pi N$ intermediate states (dashed curve) [12], $\eta N$ intermediate state (dotted curve) [13], and the $\pi \pi N$ intermediate states (dashed-dotted curve) [14]. The total sum of these contributions is shown by the full curves. The MAMI data are from ref. $[9,10]$ and the ELSA data from ref. [11].

\subsection{Linearized GDH sum rule}

Recently, it was shown that by taking derivatives of the GDH sum rule with respect to the a.m.m. one can obtain a new set of sum-rule-like relations with intriguing properties $[4,5]$.

To derive such sum rules. one begins by introducing a "classical" (or "trial") value of the particle's a.m.m., $\kappa_{0}$. At the Lagrangian level this amounts to the introduction of a Pauli term for the spin-1/2 field :

$$
\mathcal{L}_{\text {Pauli }}=\frac{i \kappa_{0}}{4 M} \bar{\psi} \sigma_{\mu \nu} \psi F^{\mu \nu}
$$



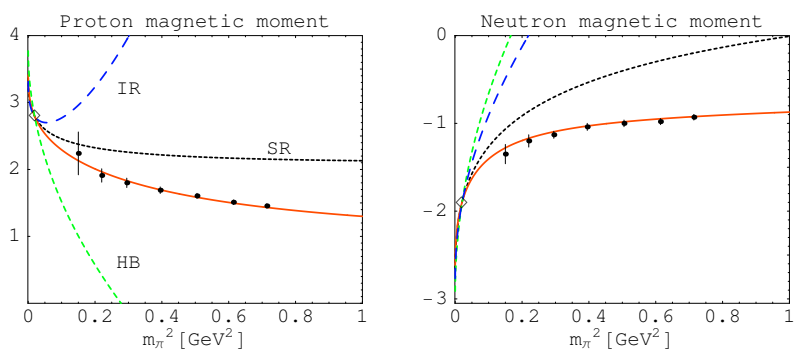

Fig. 2. Chiral behavior of proton and neutron magnetic moments (in nucleon magnetons) to one loop compared with lattice data (solid circles). "SR" (dotted lines): one-loop relativistic result based on eq. (12), "IR" (blue long-dashed lines): infrared-regularized relativistic result, "HB" (green dashed lines): leading non-analytic term in the heavy-baryon expansion. Red solid lines: single-parameter fit based on the SR result, see refs. $[4,5]$. The open diamonds represent the experimental values at the physical pion mass.

where $F^{\mu \nu}$ is the electromagnetic field tensor and $\sigma_{\mu \nu}=$ $(i / 2)\left[\gamma_{\mu}, \gamma_{\nu}\right]$ is the usual Dirac tensor operator. At the end of the calculation, $\kappa_{0}$ is set to zero, but in the evaluation of the absorption cross sections the total value of the a.m.m. is $\kappa=\kappa_{0}+\delta \kappa$, with $\delta \kappa$ denoting the loop contribution. It was shown in ref. $[4,5]$ that this yields the SR :

$$
\frac{4 \pi^{2} \alpha_{e m}}{M^{2}} \kappa=\left.\int_{0}^{\infty} \Delta \sigma^{\prime}(\nu)\right|_{\kappa_{0}=0} \frac{\mathrm{d} \nu}{\nu},
$$

where $\Delta \sigma^{\prime}(\nu)$ is the derivative of an absorption cross section w.r.t. the trial a.m.m. value $\kappa_{0}$. The striking feature of this sum rule is the linear relation between the a.m.m. and the (derivative of the) photoabsorption cross section, in contrast to the GDH SR where $\kappa$ appears quadratically. Although the cross-section quantity $\Delta \sigma^{\prime}(\nu)$ is not an observable, it is very clear how it can be determined within a specific theory. Thus, for example, the first derivative of the tree-level cross-section with respect to $\kappa_{0}$, at $\kappa_{0}=0$, in QED was worked out in ref. [4], yielding Schwinger's one-loop result. It is noteworthy that this result is reproduced by computing only a (derivative of the) tree-level Compton scattering cross-section and then performing an integration over energy. This is definitely much simpler than obtaining the Schwinger result from the GDH SR directly [15], which requires an input at the one-loop level.

The SR of eq. (12) can furthermore be applied to study the magnetic moment and polarizabilities of the nucleon in a relativistic chiral EFT framework $[4,5]$. In particular it allows to study the chiral extrapolation of these quantities, as shown in fig. 2 for the magnetic moments. One sees that the SR calculation, strictly satisfying analyticity, is better suited for the chiral extrapolation of lattice QCD results than the usual heavy-baryon expansions or the "infraredregularized" relativistic theory.

\section{Nucleon excitation spectrum}

The sum rules for Compton scattering off the nucleon are dominated by its first excited state - the $\Delta(1232)$ reso- nance, as is apparent from fig. 1. Through the sum rules, the $\Delta$ therefore plays a preponderant role in our understanding of low-energy nucleon structure. This justifies a dedicated effort to study this resonance.

High-precision measurements of the $N$-to- $\Delta$ transition by means of electromagnetic probes became possible with the advent of the new generation of electron scattering facilities, such as BATES, MAMI, and JLab, many measurements being completed in recent years $[16,17,18,19]$.

The electromagnetic nucleon-to- $\Delta$ (or, in short $\gamma N \Delta$ ) transition is predominantly of the magnetic dipole $(M 1)$ type. In a simple quark-model picture, this $M 1$ transition is described by a spin flip of a quark in the $s$-wave state. Any $d$-wave admixture in the nucleon or the $\Delta$ wavefunctions allows for the electric (E2) and Coulomb $(C 2)$ quadrupole transitions. Therefore by measuring these one is able to assess the presence of the $d$-wave components and hence quantify to which extent the nucleon or the $\Delta$ wave-function deviates from the spherical shape, i.e., to which extent they are "deformed" [20]. The $\gamma N \Delta$ transition, on the other hand, was accurately measured in the pion photo- and electro-production reactions in the $\Delta$-resonance energy region. The $E 2$ and $C 2$ transitions were found to be relatively small at moderate momentumtransfers $\left(Q^{2}\right)$, the ratios $R_{E M}=E 2 / M 1$ and $R_{S M}=$ $C 2 / M 1$ are at the level of a few percent.

Traditionally, the resonance parameters are extracted by using unitary isobar models [21,22,23,24,25,12,26], which in essence are unitarized tree-level calculations based on phenomenological Lagrangians. However, at low $Q^{2}$ the $\gamma N \Delta$-transition shows great sensitivity to the "pion cloud", which until recently could only be comprehensively studied within dynamical models [27, 28,29,30, 31,32 , which - unlike the isobar models - include quantum effects due to pion loops.

With the advent of the chiral effective field theory $(\chi \mathrm{EFT})$ of QCD $[33,34]$ and its extensions to the $\Delta(1232)$ region $[35,36,37,38,39,40]$, it has become possible to study the nucleon and $\Delta$-resonance properties in a profoundly different way. Recently, first relativistic $\chi$ EFT studies were performed of the $\gamma N \Delta$-transition in pion electroproduction $[41,42]$ and of the $\Delta(1232)$ magnetic dipole moment (MDM) in the radiative pion photoproduction [43]. The advantages over the previous dynamical approaches are apparent: $\chi \mathrm{EFT}$ is a low-energy effective field theory of QCD and as such it provides a firm theoretical foundation, with all the relevant symmetries and scales of QCD built in consistently.

The $\chi \mathrm{EFT}$ of the strong interaction is indispensable, at least at present, in relating the low-energy observables (e.g., hadron masses, magnetic moments, form factors) to $a b$ initio QCD calculations on the lattice. On the other hand, $\chi \mathrm{EFT}$ can and should be used in extracting various hadronic properties from the experiment. The $\chi \mathrm{EFT}$ fulfills both of these roles in a gratifying fashion.

The following sections review recent progress in the $\chi \mathrm{EFT}$ in the $\Delta$-resonance region that has been obtained for the $N$ and $\Delta$ masses [44], the $\gamma N \Delta$ transition [41,42], and the $\triangle \mathrm{MDM}[43]$. 


\subsection{Chiral effective field theory in the $\Delta(1232)$ region}

Starting from the effective Lagrangian of chiral perturbation theory $(\chi \mathrm{PT})$ with pion and nucleon fields [45], the $\Delta$ is included explicitly in the so-called $\delta$-expansion scheme [39]. In the following, the Lagrangian $\mathcal{L}^{(i)}$ is organized such that superscript $i$ stands for the power of electromagnetic coupling $e$ plus the number of derivatives of pion and photon fields. Writing here only the terms involving the spin-3/2 isospin-3/2 field $\Delta^{\mu}$ of the $\Delta$-isobar gives: ${ }^{1}$

$$
\begin{aligned}
\mathcal{L}_{N \Delta}^{(1)}= & \bar{\Delta}_{\mu}\left(i \gamma^{\mu \nu \alpha} D_{\alpha}-M_{\Delta} \gamma^{\mu \nu}\right) \Delta_{\nu} \\
& +\frac{i h_{A}}{2 f_{\pi} M_{\Delta}}\left\{\bar{N} T_{a} \gamma^{\mu \nu \lambda}\left(\partial_{\mu} \Delta_{\nu}\right) D_{\lambda} \pi^{a}+\text { H.c. }\right\} \\
& -\frac{H_{A}}{2 M_{\Delta} f_{\pi}} \varepsilon^{\mu \nu \rho \sigma} \bar{\Delta}_{\mu} \mathcal{T}^{a}\left(\partial_{\rho} \Delta_{\nu}\right) \partial_{\sigma} \pi^{a}, \\
\mathcal{L}_{N \Delta}^{(2)}= & \frac{i e\left(\mu_{\Delta}-1\right)}{2 M_{\Delta}} \bar{\Delta}_{\mu} \Delta_{\nu} F^{\mu \nu} \\
& +\frac{3 i e g_{M}}{2 M_{N}\left(M_{N}+M_{\Delta}\right)}\left\{\bar{N} T_{3} \partial_{\mu} \Delta_{\nu} \tilde{F}^{\mu \nu}+\text { H.c. }\right\} \\
& -\frac{e h_{A}}{2 f_{\pi} M_{\Delta}}\left\{\bar{N} T_{a} \gamma^{\mu \nu \lambda} A_{\mu} \Delta_{\nu} \partial_{\lambda} \pi^{a}+\text { H.c. }\right\}, \\
\mathcal{L}_{N \Delta}^{(3)}= & \frac{-3 e}{2 M_{N}\left(M_{N}+M_{\Delta}\right)} \bar{N} T^{3} \gamma_{5}\left[g_{E}\left(\partial_{\mu} \Delta_{\nu}\right)\right. \\
& \left.+\frac{i g_{C}}{M_{\Delta}} \gamma^{\alpha}\left(\partial_{\alpha} \Delta_{\nu}-\partial_{\nu} \Delta_{\alpha}\right) \partial_{\mu}\right] F^{\mu \nu}+\text { H.c. },
\end{aligned}
$$

where $M_{N}$ and $M_{\Delta}$ are, respectively, the nucleon and $\Delta$ isobar masses, $N$ and $\pi^{a}(a=1,2,3)$ stand for the nucleon and pion fields, $D_{\mu}$ is the covariant derivative ensuring the electromagnetic gauge-invariance, $F^{\mu \nu}$ and $\tilde{F}^{\mu \nu}$ are the electromagnetic field strength and its dual, $T_{a}$ are the isospin $1 / 2$ to $3 / 2$ transition matrices, and $\mathcal{T}^{a}$ are the generators in the isospin $3 / 2$ representation of $S U(2)$, satisfying $\mathcal{T}^{a} \mathcal{T}^{a}=5 / 3$. The coupling constants are given by : $f_{\pi}=92.4 \mathrm{MeV}, h_{A} \simeq 2.85$ is obtained from the $\Delta$ resonance width, $\Gamma_{\Delta}=0.115 \mathrm{GeV}$, and for $H_{A}$ the large$N_{c}$ relation $H_{A}=(9 / 5) g_{A}$ is adopted, with $g_{A} \simeq 1.267$ the nucleon axial-coupling constant.

Note that the electric and the Coulomb $\gamma N \Delta$ couplings ( $g_{E}$ and $g_{C}$, respectively) are of one order higher than the magnetic $\left(g_{M}\right)$ one, because of the $\gamma_{5}$ which involves the "small components" of the fermion fields and thus introduces an extra power of the 3 -momentum. The MDM $\mu_{\Delta}$ is defined here in units of $\left[e / 2 M_{\Delta}\right]$. Higher electromagnetic moments are omitted, because they do not contribute at the orders that we consider.

Note that $\mathcal{L}_{\Delta}^{(1)}$ contains the free Lagrangian, which is formulated in [46] such that the number of spin degrees of freedom of the relativistic spin- $3 / 2$ field is constrained to the physical number: $2 s+1=4$. The $N$ to $\Delta$ transition couplings in eqs. $(13,14,15)$ are consistent with these constraints $[47,48,49]$. The $\gamma \Delta \Delta$ coupling is more subtle since

\footnotetext{
${ }^{1}$ Here we introduce totally antisymmetric products of $\gamma$ matrices: $\gamma^{\mu \nu}=\frac{1}{2}\left[\gamma^{\mu}, \gamma^{\nu}\right], \gamma^{\mu \nu \alpha}=\frac{1}{2}\left\{\gamma^{\mu \nu}, \gamma^{\alpha}\right\}=i \varepsilon^{\mu \nu \alpha \beta} \gamma_{\beta} \gamma_{5}$.
}

in this case constraints do not hold for sufficiently strong electromagnetic fields, see, e.g., [50]. In extracting the $\Delta$ $\mathrm{MDM}$, it is therefore assumed that the electromagnetic field is weak, compared to the $\Delta$ mass scale.

The inclusion of the $\Delta$-resonance introduces another light scale - besides the pion mass - in the theory, the resonance excitation energy: $\Delta \equiv M_{\Delta}-M_{N} \sim 0.3 \mathrm{GeV}$. This energy scale is still relatively light in comparison to the chiral symmetry breaking scale $\Lambda_{\chi S B} \sim 1 \mathrm{GeV}$. Therefore, $\delta=\Delta / \Lambda_{\chi S B}$ can be treated as a small parameter. The question is, how to compare this parameter with the small parameter of chiral perturbation theory $(\chi \mathrm{PT})$, $\epsilon=m_{\pi} / \Lambda_{\chi S B}$.

In most of the literature (see, e.g., refs. [35,36,37,38, 40]) they are assumed to be of comparable size, $\delta \approx \epsilon$. This, however, leads to a somewhat unsatisfactory result because obviously the $\Delta$-contributions are overestimated at lower energies and underestimated at the resonance energies. To estimate the $\Delta$-resonance contributions correctly, and depending on the energy region, one needs to count $\delta$ and $\epsilon$ differently.

A relation $\epsilon=\delta^{2}$ was suggested and explored in [39], and is referred to as the $\delta$-expansion. The second power is indeed the closest integer power for the relation of these parameters in the real world. In refs. $[44,41,42,43]$ this relation was used for power-counting purposes only, and was not imposed in the actual evaluations of diagrams. Each diagram is simply characterized by an overall $\delta$-counting index $n$, which tells us that its contribution begins at $\mathrm{O}\left(\delta^{n}\right)$

Because of the distinction of $m_{\pi}$ and $\Delta$ the counting of a given diagram depends on whether the characteristic momentum $p$ is in the low-energy region $\left(p \sim m_{\pi}\right)$ or in the resonance region $(p \sim \Delta)$. In the low-energy region the index of a graph with $L$ loops, $N_{\pi}$ pion propagators, $N_{N}$ nucleon propagators, $N_{\Delta} \Delta$-propagators, and $V_{i}$ vertices of dimension $i$ is

$n=2\left(\sum_{i} i V_{i}+4 L-N_{N}-2 N_{\pi}\right)-N_{\Delta} \equiv 2 n_{\chi \mathrm{PT}}-N_{\Delta}$,

where $n_{\chi \mathrm{PT}}$ is the index in $\chi \mathrm{PT}$ with no $\Delta$ 's [45]. In the resonance region, one distinguishes the one- $\Delta$-reducible $(\mathrm{O} \Delta \mathrm{R})$ graphs [39]. Such graphs contain $\Delta$ propagators which go as $1 /(p-\Delta)$, and hence for $p \sim \Delta$ they are large and all need to be included. This gives an incentive, within the power-counting scheme, to resum $\Delta$ contributions. Their resummation amounts to dressing the $\Delta$ propagators so that they behave as $1 /(p-\Delta-\Sigma)$. The self-energy $\Sigma$ begins at order $p^{3}$ and thus a dressed $\mathrm{O} \Delta \mathrm{R}$ propagator counts as $1 / \delta^{3}$. If the number of such propagators in a graph is $N_{O \Delta R}$, the power-counting index of this graph in the resonance region is given by

$$
n=n_{\chi \mathrm{PT}}-N_{\Delta}-2 N_{O \Delta R},
$$

where $N_{\Delta}$ is the total number of $\Delta$-propagators.

A word on the renormalization program, as it is an indivisible part of power counting in a relativistic theory. Indeed, without some kind of renormalization the loop 
graphs diverge as $\Lambda^{\mathcal{N}}$, where $\Lambda$ is an ultraviolet cutoff, and $\mathcal{N}$ is a positive power proportional to the powercounting index of the graph. Also, contributions of heavy scales, such as baryon masses, may appear as $M^{\mathcal{N}}$. The renormalization of the loop graphs can and should be performed so as to absorb these large contributions into the available low-energy constants, thus bringing the result in accordance with power counting [51].

To give an example, consider the one- $\pi N$-loop contribution to the nucleon mass. For the $\pi N N$ vertex, the power counting tells us that this contribution begins at $O\left(m_{\pi}^{3}\right)$. An explicit calculation, however, will show (e.g., [45]) that the loop produces $O\left(m_{\pi}^{0}\right)$ and $O\left(m_{\pi}^{2}\right)$ terms, both of which are (infinitely) large. This is not a violation of power counting, because there are two lowenergy constants: the nucleon mass in the chiral limit, $M^{(0)}$, and $c_{1 N}$, which enter at order $O\left(m_{\pi}^{0}\right)$ and $O\left(m_{\pi}^{2}\right)$, respectively, and renormalize away the large contributions coming from the loop. The renormalized relativistic result, up to and including $O\left(m_{\pi}^{3}\right)$, can be written as [44]:

$$
\begin{aligned}
M_{N}= & M_{N}^{(0)}-4 c_{1 N} m_{\pi}^{2} \\
& -\frac{3 g_{A}^{2}}{\left(8 \pi f_{\pi}\right)^{2}} m_{\pi}^{3}\left\{4\left(1-\frac{m_{\pi}^{2}}{4 M_{N}^{2}}\right)^{5 / 2} \arccos \frac{m_{\pi}}{2 M_{N}}\right. \\
& +\frac{17 m_{\pi}}{16 M_{N}}-\left(\frac{m_{\pi}}{2 M_{N}}\right)^{3} \\
& \left.+\frac{m_{\pi}}{8 M_{N}}\left[30-10\left(\frac{m_{\pi}}{M_{N}}\right)^{2}+\left(\frac{m_{\pi}}{M_{N}}\right)^{4}\right] \ln \frac{m_{\pi}}{M_{N}}\right\},
\end{aligned}
$$

and one can easily verify that the loop contribution begins at $O\left(m_{\pi}^{3}\right)$ in agreement with power counting.

Likewise, the $\Delta$ mass has also been calculated in relativistic $\chi \mathrm{EFT}$ see ref. [44] for details.

The $m_{\pi}$ dependence of the nucleon and $\Delta$-resonance masses are compared with lattice results in fig. 3. One of the two parameters in eq. (18) is constrained by the physical nucleon mass value at $m_{\pi}=0.139 \mathrm{GeV}$, while the other parameter is fit to the lattice data shown in the figure. This yields : $M_{N}^{(0)}=0.883 \mathrm{GeV}$ and $c_{1 N}=-0.87 \mathrm{GeV}^{-1}$. As is seen from the figure, with this two-parameter form for $M_{N}$, a good description of lattice results is obtained up to $m_{\pi}^{2} \simeq 0.5 \mathrm{GeV}^{2}$. Analogously to the nucleon case, one low-energy constant for the $\Delta$ is fixed from the physical value of the $\Delta$ mass, while the second parameter is fit to the lattice data shown in fig. 3, yielding : $M_{\Delta}^{(0)}=1.20 \mathrm{GeV}$ and $c_{1 \Delta}=-0.40 \mathrm{GeV}^{-1}$. As well as for the nucleon, this two-parameter form for $M_{\Delta}$ yields a fairly good description of the lattice results up to $m_{\pi}^{2} \simeq 0.5 \mathrm{GeV}^{2}$.

\section{$3.2 \gamma \mathrm{N} \Delta$ transition}

The $\gamma N \Delta$ transition is usually studied through the pion electroproduction process. The pion electroproduction amplitude to NLO in the $\delta$ expansion, in the resonance region, is given by graphs in fig. 4(a) and (b), where the shaded blobs in graph (a) include corrections depicted in

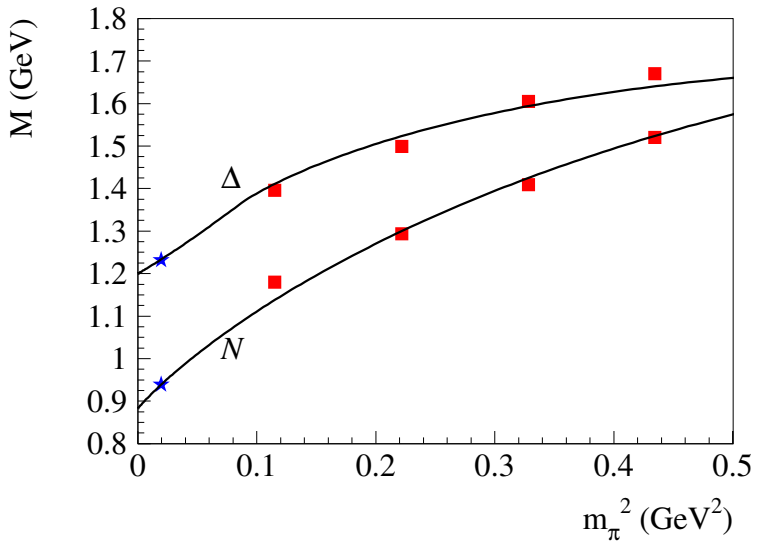

Fig. 3. Pion-mass dependence of the nucleon and $\Delta(1232)$ masses. The curves are two-parameter expressions for the $\pi N$ loop contributions to $M_{N}$ and $M_{\Delta}$ as calculated in ref. [44] (see text for the values of the low-energy constants). The red squares are lattice results from the MILC Collaboration [52]. The stars represent the physical mass values.

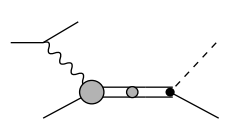

(a)

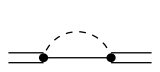

(c)

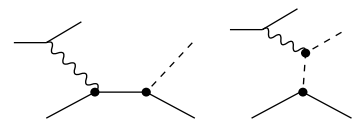

(b)

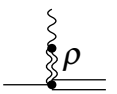

(d)

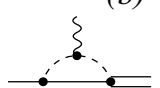

(e)
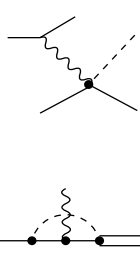

(f)
Fig. 4. Diagrams for the $e N \rightarrow e \pi N$ reaction at NLO in the $\delta$-expansion. Double lines represent the $\Delta$ propagators.

fig. 4(c-f). The hadronic part of graph (a) begins at $\mathcal{O}\left(\delta^{0}\right)$ which here is the leading order. The Born graphs (b) contribute at $\mathcal{O}(\delta)$. The one-loop vertex corrections of fig. 4(e) and (f) to the $\gamma N \Delta$-transition form factors have been evaluated in two independent ways in refs. [41,42], to which we refer for details. At NLO there are also vertex corrections of the type (e) and (f) with nucleon propagators in the loop replaced by the $\Delta$-propagators. However, after the appropriate renormalizations and $Q^{2} \ll \Lambda_{\chi S B} \Delta$, these graphs start to contribute at next-next-to-leading order. The vector-meson diagram, fig. 4(d), contributes to NLO for $Q^{2} \sim \Lambda_{\chi S B} \Delta$. It was included effectively in refs. [41,42] by giving the $g_{M}$-term a dipole $Q^{2}$-dependence, in analogy to how it is usually done for the nucleon isovector form factor.

The resonant pion photoproduction multipoles are used to determine the two low-energy constants: $g_{M}$ and $g_{E}$, the strength of the $M 1$ and $E 2 \gamma N \Delta$ transitions. In fig. 5, we show the result of the $\chi \mathrm{EFT}$ calculations for the pion photoproduction resonant multipoles $M_{1+}^{(3 / 2)}$ and $E_{1+}^{(3 / 2)}$, around the resonance position, as function of the total c.m. energy $W$ of the $\pi N$ system. These two multipoles are well established by the MAID [12] and SAID [53] partial-wave solutions which allow us to fit 

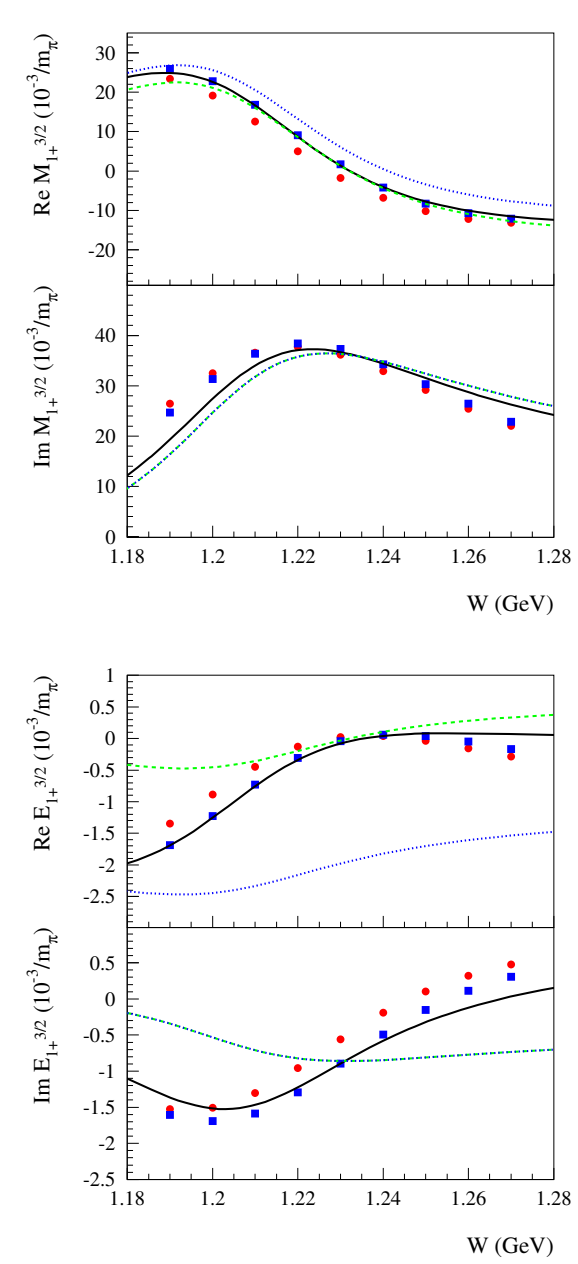

Fig. 5. Multipole amplitudes $M_{1+}^{(3 / 2)}$ (top panels) and $E_{1+}^{(3 / 2)}$ (bottom panels) for pion photoproduction as function of the invariant mass $W$ of the $\pi N$ system. Dashed curves: $\Delta$ contribution without the $\gamma N \Delta$-vertex corrections, (i.e., fig. 4(a) without fig. $4(\mathrm{e}, \mathrm{f}))$. Dotted curves: adding the Born contributions, fig. 4(b), to the dashed curves. Solid curves: complete NLO calculation, includes all graphs from fig. 4. In all curves the low-energy parameters are chosen as : $g_{M}=2.9, g_{E}=-1.0$. The data point are from the SAID analysis (FA04K) [53] (red circles), and from the MAID 2003 analysis [12] (blue squares).

the two low-energy constants of the chiral Lagrangian of eqs. $(14,15)$ as : $g_{M}=2.9, g_{E}=-1.0$. As is seen from fig. 5 , with these values the NLO results (solid lines) give a good description of the energy dependence of the resonant multipoles in a window of $100 \mathrm{MeV}$ around the $\Delta$ resonance position. Also, these values yield $R_{E M}=-2.3$ $\%$, in a nice agreement with experiment [16].

The dashed curves in fig. 5 show the contribution of the $\Delta$-resonant diagram of fig. 4(a) without the NLO vertex corrections fig. $4(\mathrm{e}, \mathrm{f})$. For the $M_{1+}$ multipole this is the LO contribution. For the $E_{1+}$ multipole the LO contribution is absent (the $g_{E}$ coupling is of one order higher than $\left.g_{M}\right)$. Hence, the dashed curve represents a partial NLO contribution to $E_{1+}$ therein. Upon adding the nonresonant Born graphs, fig. 4(b), to the dashed curves one
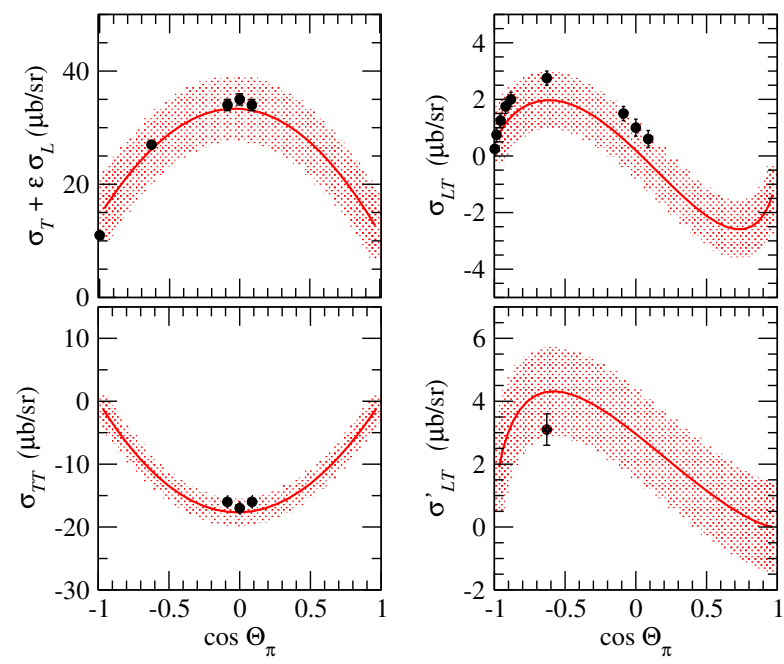

Fig. 6. $\chi$ EFT NLO results for the $\Theta_{\pi}$ dependence of the $\gamma^{*} p \rightarrow$ $\pi^{0} p$ cross sections at $W=1.232 \mathrm{GeV}$ and $Q^{2}=0.127 \mathrm{GeV}^{2}$. The theoretical error bands are described in the text. Data points are from BATES experiments $[18,54]$.

obtains the dotted curves in fig. 5. These non-resonant contributions are purely real at this order and do not affect the imaginary part of the multipoles. One sees that the resulting calculation is flawed because the real parts of the resonant multipoles now fail to cross zero at the resonance position and hence unitarity, in the sense of Watson's theorem [55], is violated. The complete NLO calculation, shown by the solid curves in fig. 5 , includes in addition the vertex corrections, fig. 4(e, f), which restore unitarity exactly. Watson's theorem is satisfied exactly by the $N L O$, up to-one-loop amplitude given the graphs in fig. 4 .

Figure 6 shows the NLO results for different virtual photon absorption cross sections (for definitions, see ref. [42]) at the resonance position, and for $Q^{2} \simeq$ $0.127 \mathrm{GeV}^{2}$, where recent precision data are available. Besides the low-energy constants $g_{M}$ and $g_{E}$, which were fixed from the resonant multipoles in fig. 5 , the only other low-energy constant from eq. (15) entering the NLO electroproduction calculation is $g_{C}$. The main sensitivity on $g_{C}$ enters in $\sigma_{L T}$. A best description of the $\sigma_{L T}$ data in fig. 6 is obtained by choosing $g_{C}=-2.36$.

The theoretical uncertainty due to the neglect of higher-order effects was estimated in ref. [42]. We know that they must be suppressed by at least one power of $\delta$ $\left(=\Delta / \Lambda_{\chi S B}\right)$ as compared to the NLO and two powers of $\delta$ as compared to the LO contributions. These error estimates are shown by the bands in fig. 6 . One sees that the NLO $\chi$ EFT calculation, within its accuracy, is consistent with the experimental data for these observables.

Figure 7 shows the $Q^{2}$ dependence of the ratios $R_{E M}$ and $R_{S M}$. Having fixed the low energy constants $g_{M}, g_{E}$ and $g_{C}$, this $Q^{2}$ dependence follows as a prediction. The theoretical uncertainty here (shown by the error bands) was also estimated in ref. [42] over the range of $Q^{2}$ from 0 to $0.2 \mathrm{GeV}^{2}$. One sees that the NLO calculations are consistent with the experimental data for both of the ratios. 


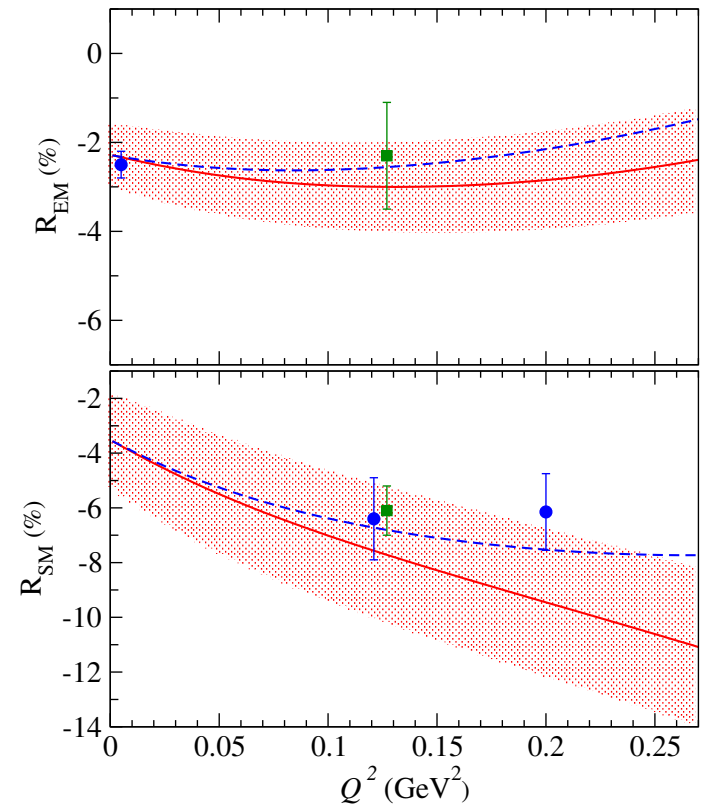

Fig. 7. $Q^{2}$ dependence of the NLO results (solid curves) for $R_{E M}$ (upper panel) and $R_{S M}$ (lower panel) [41,42]. The blue dashed curves represent a phenomenological estimate of $\mathrm{N}^{2} \mathrm{LO}$ effects by including $Q^{2}$-dependence in $g_{E}$ according to a dipole behavior, see ref. [42]. The blue circles are data points from MAMI for $R_{E M}$ [16], and $R_{S M}[56,57]$. The green squares are data points from BATES [18].

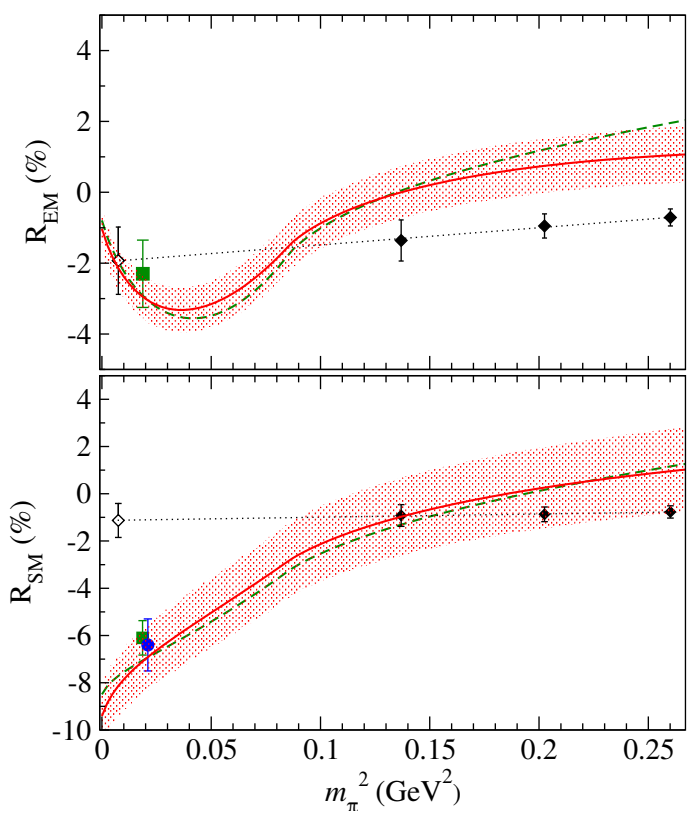

Fig. 8. $m_{\pi}$ dependence of the NLO results at $Q^{2}=0.1 \mathrm{GeV}^{2}$ for $R_{E M}$ (upper panel) and $R_{S M}$ (lower panel). The blue circle is a data point from MAMI [56], the green squares are data points from BATES [18]. The three filled black diamonds at larger $m_{\pi}$ are lattice calculations [58], whereas the open diamond near $m_{\pi} \simeq 0$ represents their extrapolation assuming linear dependence in $m_{\pi}^{2}$. Solid curves: NLO result when accounting for the $m_{\pi}$ dependence in $M_{N}$ and $M_{\Delta}$; Dashed curves: NLO result of ref. [41], where the $m_{\pi}$-dependence of $M_{N}$ and $M_{\Delta}$ was not accounted for.
Figure 8 shows the $m_{\pi}$-dependence of the ratios $R_{E M}$ and $R_{S M}$ and compares them to lattice QCD calculations. The recent state-of-the-art lattice calculations of $R_{E M}$ and $R_{S M}$ [58] use a linear, in the quark mass $\left(m_{q} \propto m_{\pi}^{2}\right)$, extrapolation to the physical point, thus assuming that the non-analytic $m_{q}$-dependencies are negligible. The thus obtained value for $R_{S M}$ at the physical $m_{\pi}$ value displays a large discrepancy with the experimental result, as seen in fig. 8. The relativistic $\chi \mathrm{EFT}$ calculation, on the other hand, shows that the non-analytic dependencies are not negligible. While at larger values of $m_{\pi}$, where the $\Delta$ is stable, the ratios display a smooth $m_{\pi}$ dependence, at $m_{\pi}=\Delta$ there is an inflection point, and for $m_{\pi} \leq \Delta$ the non-analytic effects are crucial.

One also notices from fig. 8 that there is only little difference between the $\chi \mathrm{EFT}$ calculations with the $m_{\pi^{-}}$ dependence of $M_{N}$ and $M_{\Delta}$ accounted for, and an earlier calculation [41], where the ratios were evaluated neglecting the $m_{\pi}$-dependence of the masses.

Figure 8 also shows a theoretical uncertainty of the ratios $R_{E M}$ and $R_{S M}$ taken over the range of $m_{\pi}^{2}$ from 0 to $0.15 \mathrm{GeV}^{2}$. The $m_{\pi}$ dependence obtained from $\chi \mathrm{EFT}$ clearly shows that the lattice results for $R_{S M}$ may in fact be consistent with experiment.

\section{$3.3 \Delta(1232)$ magnetic dipole moment}

Although the $\Delta(1232)$-isobar is the most distinguished and well-studied nucleon resonance, such a fundamental property as its magnetic dipole moment (MDM) has thusfar escaped a precise determination. The problem is generic to any unstable particle whose lifetime is too short for its MDM to be measurable in the usual way through spin precession experiments. A measurement of the MDM of such an unstable particle can apparently be done only indirectly, in a three-step process, where the particle is first produced, then emits a low-energy photon which plays the role of an external magnetic field, and finally decays. In this way the MDM of $\Delta^{++}$is accessed in the reaction $\pi^{+} p \rightarrow \pi^{+} p \gamma[59,60]$ while the MDM of $\Delta^{+}$can be determined using the radiative pion photoproduction $\left(\gamma p \rightarrow \pi^{0} p \gamma^{\prime}\right)[61]$.

A first experiment devoted to the MDM of $\Delta^{+}$was completed in 2002 [62]. The value extracted in this experiment, $\mu_{\Delta^{+}}=2.7_{-1.3}^{+1.0}$ (stat.) \pm 1.5 (syst.) \pm 3 (theor.) $($ nuclear magnetons), is based on theoretical input from the phenomenological model $[63,64]$ of the $\gamma p \rightarrow \pi^{0} p \gamma^{\prime}$ reaction. To improve upon the precision of this measurement, a dedicated series of experiments has recently been carried out by the Crystal Ball Collaboration at MAMI [65]. These experiments achieve about two orders of magnitude better statistics than the pioneering experiment [62]. The aim of the investigation within $\chi \mathrm{EFT}$ was to complement these high-precision measurements with an accurate and model-independent analysis of the $\gamma p \rightarrow \pi^{0} p \gamma^{\prime}$ reaction.

The optimal sensitivity of the $\gamma p \rightarrow \pi^{0} p \gamma^{\prime}$ reaction to the MDM term is achieved when the incident photon energy is in the vicinity of $\Delta$, while the outgoing photon 


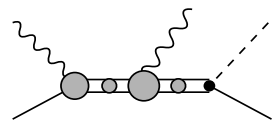

(a)

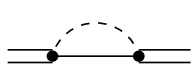

(d)

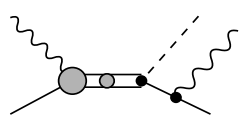

(b)

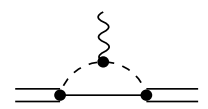

(e)

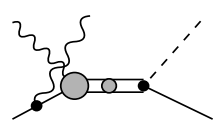

(c)

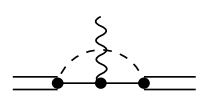

(f)
Fig. 9. Diagrams for the $\gamma p \rightarrow \pi^{0} p \gamma^{\prime}$ reaction at NLO in the $\delta$-expansion, considered in this work. Double lines represent the $\Delta$ propagators.

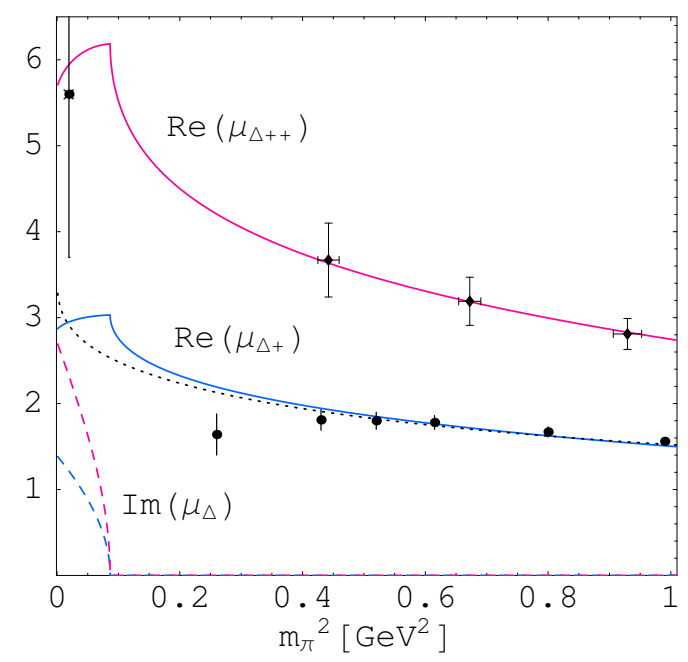

Fig. 10. Pion mass dependence of the real (solid curves) and imaginary (dashed curves) parts of $\Delta^{++}$and $\Delta^{+}$MDMs (in nuclear magnetons). Dotted curve is the result for the proton magnetic moment from ref. [4]. The experimental data point for $\Delta^{++}$is from PDG analysis [66]. Lattice data are from [67] for $\Delta^{++}$and from [68] for $\Delta^{+}$.

energy is of order of $m_{\pi}$. In this case the $\gamma p \rightarrow \pi^{0} p \gamma^{\prime}$ amplitude to next-to-leading order (NLO) in the $\delta$-expansion is given by the diagrams of fig. $9(\mathrm{a}-\mathrm{c})$, where the shaded blobs, in addition to vertices from eqs. $(13,14,15)$, contain the one-loop corrections shown in fig. $9(\mathrm{~d}-\mathrm{f})$. The contributions to $\mu_{\Delta}$ of diagrams $(e)$ and $(f)$ in fig. 9 have been calculated in ref. [43], to which we refer for technical details. The evaluation of these loop diagrams also allows to quantify the $m_{\pi}$ dependence of $\mu_{\Delta}$ which can be used to compare with lattice QCD results. As all lattice data for $\mu_{\Delta}$ at present and in the foreseeable future are for larger than the physical values of $m_{\pi}$, their comparison with experiment requires the knowledge of the $m_{\pi}$-dependence for this quantity. Figure 10 shows the pion mass dependence of real and imaginary parts of the $\Delta^{+}$and $\Delta^{++}$ MDMs, according to our one-loop calculation. Each of the two solid curves has a free parameter, the counterterm $\mu_{\Delta}$ from $\mathcal{L}_{N \Delta}^{(2)}$, adjusted to agree with the lattice data at larger values of $m_{\pi}$. As can be seen from fig. 10, the $\Delta \mathrm{MDM}$ develops an imaginary part when $m_{\pi}<\Delta=M_{\Delta}-M$, whereas the real part has a pronounced cusp at $m_{\pi}=\Delta$.
For $\mu_{\Delta^{+}}$, the curve is in disagreement with the trend of the recent lattice data, which possibly is due to the "quenching" in the lattice calculations. The dotted line in fig. 10 shows the result [4] for the magnetic moment for the proton. One sees that $\mu_{\Delta^{+}}$and $\mu_{p}$, while having very distinct behavior for small $m_{\pi}$, are approximately equal for larger values of $m_{\pi}$.

We next discuss the $\chi$ EFT results for the $\gamma p \rightarrow \pi^{0} p \gamma^{\prime}$ observables. The NLO calculation of this process in the $\delta$ expansion corresponds with the diagrams of fig. 9. This calculation completely fixes the imaginary part of the $\gamma \Delta \Delta$ vertex. It leaves $\mu_{\Delta}$ as only free parameter, which enters as a low energy constant in $\mathcal{L}_{N \Delta}^{(2)}$. Thus the real part of $\mu_{\Delta^{+}}$is to be extracted from the $\gamma p \rightarrow \pi^{0} p \gamma^{\prime}$ observables, some of which are shown in fig. 11 for an incoming photon energy $E_{\gamma}^{l a b}=400 \mathrm{MeV}$ as function of the emitted photon energy $E_{\gamma}^{\prime c . m .}$. In the soft-photon limit $\left(E_{\gamma}^{\prime c . m .} \rightarrow 0\right)$, the $\gamma p \rightarrow \pi^{0} p \gamma^{\prime}$ reaction is completely determined from the bremsstrahlung process from the initial and final protons. The deviations of the $\gamma p \rightarrow \pi^{0} p \gamma^{\prime}$ observables, away from the soft-photon limit, will then allow to study the sensitivity to $\mu_{\Delta^{+}}$. It is therefore very useful to introduce the ratio [64]:

$$
R \equiv \frac{1}{\sigma_{\pi}} \cdot E_{\gamma}^{\prime} \frac{\mathrm{d} \sigma}{\mathrm{d} E_{\gamma}^{\prime}}
$$

where $\mathrm{d} \sigma / \mathrm{d} E_{\gamma}^{\prime}$ is the $\gamma p \rightarrow \pi^{0} p \gamma^{\prime}$ cross section integrated over the pion and photon angles, and $\sigma_{\pi}$ is the angular integrated cross section for the $\gamma p \rightarrow \pi^{0} p$ process weighted with the bremsstrahlung factor, as detailed in [64]. This ratio $R$ has the property that in the soft-photon limit, the low energy theorem predicts that $R \rightarrow 1$. From fig. 11 one then sees that the $\chi \mathrm{EFT}$ calculation obeys this theorem. This is a consequence of gauge-invariance which is maintained exactly throughout the calculation, also away from the soft-photon limit.

The $\chi$ EFT result for $R$ shows clear deviations from unity at higher outgoing photon energies, in good agreement with the first data for this process [62]. The sensitivity of the $\chi \mathrm{EFT}$ calculation to the $\mu_{\Delta}$ is a very promising setting for the dedicated second-generation experiment which has recently been completed by the Crystal Ball Coll. at MAMI [65]. It improves upon the statistics of the first experiment (fig. 11) by at least two orders of magnitude and will allow for a reliable extraction of $\mu_{\Delta^{+}}$using the $\chi \mathrm{EFT}$ calculation presented here.

Besides the cross section, the $\gamma p \rightarrow \pi^{0} p \gamma^{\prime}$ asymmetries for linearly and circularly polarized incident photons have also been measured in the recent dedicated experiment [65]. They are also shown in fig. 11. The photon asymmetry for linearly polarized photons, $\Sigma$, at $E_{\gamma}^{\prime}=0$ exactly reduces to the $\gamma p \rightarrow \pi^{0} p$ asymmetry. It is seen from fig. 11 that the $\chi \mathrm{EFT}$ calculation is in good agreement with the experimental value. At higher outgoing photon energies, the photon asymmetry as predicted by the NLO $\chi$ EFT calculation remains nearly constant and is very weakly dependent on $\mu_{\Delta}$. It is an ideal observable for a consistency check of the $\chi \mathrm{EFT}$ calculation and to test that the $\Delta$ diagrams of fig. 9 indeed dominate the 


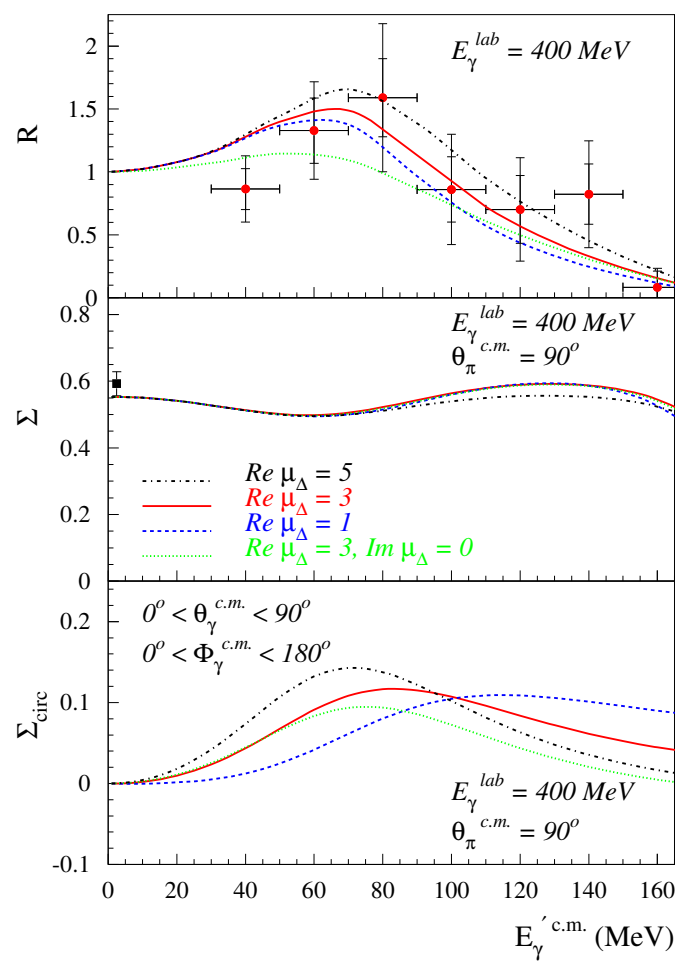

Fig. 11. The outgoing photon energy dependence of the $\gamma p \rightarrow \pi^{0} p \gamma^{\prime}$ observables for different values of $\mu_{\Delta^{+}}$(in units $\left.e / 2 M_{\Delta}\right)$. Top panel: the ratio of $\gamma p \rightarrow \pi^{0} p \gamma^{\prime}$ to $\gamma p \rightarrow \pi^{0} p$ crosssections eq. (19). Data points are from [62]. Middle panel: the linear-polarization photon asymmetry of the $\gamma p \rightarrow \pi^{0} p \gamma^{\prime}$ crosssections differential w.r.t. the outgoing photon energy and pion c.m. angle. The data point at $E_{\gamma}^{\prime}=0$ corresponds with the $\gamma p \rightarrow \pi^{0} p$ photon asymmetry from [16]. Lower panel: the circular-polarization photon asymmetry (as defined in [64]), where the outgoing photon angles have been integrated over the indicated range.

process. Mechanisms involving $\pi$-photoproduction Born terms followed by $\pi N$ rescattering have been considered in model calculations $[63,64]$. In the $\delta$-counting they start contributing at next-next-to-leading order and therefore will provide the main source of corrections to the present NLO results.

The asymmetry for circularly polarized photons, $\Sigma_{\text {circ }}$, (which is exactly zero for a two-body process due to reflection symmetry w.r.t. the reaction plane) has been proposed [64] as a unique observable to enhance the sensitivity to $\mu_{\Delta}$. Indeed, in the soft-photon limit, where the $\gamma p \rightarrow \pi^{0} p \gamma^{\prime}$ process reduces to a two-body process, $\Sigma_{\text {circ }}$ is exactly zero. Therefore, its value at higher outgoing photon energies is directly proportional to $\mu_{\Delta}$. One sees from fig. 11 (lower panel) that our $\chi \mathrm{EFT}$ calculation supports this observation, and shows sizeably different asymmetries for different values of $\mu_{\Delta}$. A combined fit of all three observables shown in fig. 11 will therefore allow for a very stringent test of the $\chi \mathrm{EFT}$ calculation, which can then be used to extract the $\Delta^{+}$MDM.

\section{Conclusions}

It was discussed here how Compton scattering sum rules relate low-energy nucleon structure quantities to the nucleon excitation spectrum, with special emphasis on the GDH sum rule. I demonstrated the utility of taking derivatives of the GDH sum rule, in order to convert it to forms which are sometimes more calculationally robust. In particular it was shown how it allows to estimate the chiral extrapolations of lattice QCD results for anomalous magnetic moments of nucleons.

Subsequently, new developments in our description of the nucleon excitation spectrum were discussed. In particular I reviewed recent work on a $\chi \mathrm{EFT}$ framework for the $\Delta(1232)$-resonance region. This framework plays a dual role, in that it allows for an extraction of resonance parameters from observables and predicts their $m_{\pi}$ dependence. In this way it may provide a crucial connection of present lattice QCD results obtained at unphysical values of $m_{\pi}$ to the experiment. This was demonstrated here explicitely for the $N$ and $\Delta$ masses, the $\gamma N \Delta$ transition and the $\Delta$ magnetic dipole moment. As the next-generation lattice calculations of these quantities are on the way [69], such a $\chi$ EFT framework will, hopefully, complement these efforts.

I am grateful to my colleagues in Mainz for the unique culture of "cross-fertilization" between experiment and theory. On the subject of two-photon physics, I like to thank in particular Dieter Drechsel and Barbara Pasquini, for the many collaborations. I also like to acknowledge Vladimir Pascalutsa for very fruitful recent collaborations on the $\chi \mathrm{EFT}$ in the $\Delta$-resonance region. This work is supported in part by DOE grant no. DEFG02-04ER41302 and contract DE-AC05-84ER-40150 under which SURA operates the Jefferson Laboratory.

\section{References}

1. D. Drechsel, B. Pasquini, M. Vanderhaeghen, Phys. Rep. 378, 99 (2003).

2. S.B. Gerasimov, Sov. J. Nucl. Phys. 2, 430 (1966) (Yad. Fiz. 2, 598 (1966)); S.D. Drell, A.C. Hearn, Phys. Rev. Lett. 16, 908 (1966).

3. D. Drechsel, L. Tiator, Annu. Rev. Nucl. Part. Sci. 54, 69 (2004).

4. V. Pascalutsa, B.R. Holstein, M. Vanderhaeghen, Phys. Lett. B 600, 239 (2004).

5. B.R. Holstein, V. Pascalutsa, M. Vanderhaeghen, Phys. Rev. D 72, 094014 (2005).

6. A.M. Baldin, Nucl. Phys. 18, 310 (1960).

7. L.I. Lapidus, Sov. Phys. JETP 16, 964 (1963).

8. B.R. Holstein, D. Drechsel, B. Pasquini, M. Vanderhaeghen, Phys. Rev. C 61, 034316 (2000).

9. GDH and A2 Collaborations (J. Ahrens et al.), Phys. Rev. Lett. 84, 5950 (2000).

10. GDH and A2 Collaborations (J. Ahrens et al.), Phys. Rev. Lett. 87, 022003 (2001).

11. GDH Collaboration (H. Dutz et al.), Phys. Rev. Lett. 91, 192001 (2003). 
12. D. Drechsel, O. Hanstein, S.S. Kamalov, L. Tiator, Nucl. Phys. A 645, 145 (1999).

13. D. Drechsel, S.S. Kamalov, L. Tiator, Phys. Rev. D 63, 114010 (2001).

14. H. Holvoet, PhD Thesis, University Gent (2001).

15. D.A. Dicus, R. Vega, Phys. Lett. B 501, 44 (2001).

16. R. Beck et al., Phys. Rev. Lett. 78, 606 (1997); Phys. Rev. C 61, 035204 (2000).

17. G. Blanpied et al., Phys. Rev. Lett. 79, 4337 (1997).

18. C. Mertz et al., Phys. Rev. Lett. 86, 2963 (2001); N.F. Sparveris et al., Phys. Rev. Lett. 94, 022003 (2005).

19. V.V. Frolov et al., Phys. Rev. Lett. 82, 45 (1999); K. Joo et al., Phys. Rev. Lett. 88, 122001 (2002).

20. S.L. Glashow, Physica A 96, 27 (1979); N. Isgur, G. Karl, R. Koniuk, Phys. Rev. D 25, 2394 (1982); S. Capstick, G. Karl, Phys. Rev. D 41, 2767 (1990); S. Capstick, B.D. Kiester, Phys. Rev. D 51, 3598 (1995); G.A. Miller, Phys. Rev. C 68, 022201(R) (2003); A.M. Bernstein, Eur. Phys. J. A 17, 349 (2003).

21. M.G. Olsson, E.T. Osypowski, Nucl. Phys. B 87, 399 (1974); Phys. Rev. D 17, 174 (1978); R.M. Davidson, N.C. Mukhopadhyay, R.S. Wittman, Phys. Rev. D 43, 71 (1991).

22. H. Garcilazo, E. Moya de Guerra, Nucl. Phys. A 562, 521 (1993); C. Fernandez-Ramirez, E. Moya de Guerra, J.M. Udias, arXiv:nucl-th/0509020.

23. M. Vanderhaeghen, K. Heyde, J. Ryckebusch, M. Waroquier, Nucl. Phys. A 595, 219 (1995).

24. V. Pascalutsa, O. Scholten, Nucl. Phys. A 591, 658 (1995); O. Scholten, A.Y. Korchin, V. Pascalutsa, D. Van Neck, Phys. Lett. B 384, 13 (1996); A.Y. Korchin, O. Scholten, R.G.E. Timmermans, Phys. Lett. B 438, 1 (1998).

25. T. Feuster, U. Mosel, Phys. Rev. C 59, 460 (1999); G. Penner, U. Mosel, Phys. Rev. C 66, 055211 (2002); H. Lenske, V. Shklyar, U. Mosel, arXiv:nucl-th/0512044.

26. I.G. Aznauryan, Phys. Rev. C 68, 065204 (2003).

27. S. Nozawa, B. Blankleider, T.-S.H. Lee, Nucl. Phys. A 513 , 459 (1990); T.-S.H. Lee, B.C. Pearce, Nucl. Phys. A 530, 532 (1991); T. Sato, T.-S.H. Lee, Phys. Rev. C 54, 2660 (1996); 63, 055201 (2001).

28. Y. Surya, F. Gross, Phys. Rev. C 53, 2422 (1996); 47, 703 (1993).

29. S.S. Kamalov, S.N. Yang, Phys. Rev. Lett. 83, 4494 (1999); S.S. Kamalov, G.Y. Chen, S.N. Yang, D. Drechsel, L. Tiator, Phys. Lett. B 522, 27 (2001); L. Tiator, D. Drechsel, S.S. Kamalov, S.N. Yang, Eur. Phys. J. A 17, 357 (2003).

30. M.G. Fuda, H. Alharbi, Phys. Rev. C 68, 064002 (2003).

31. V. Pascalutsa, J.A. Tjon, Phys. Lett. B 435, 245 (1998); Phys. Rev. C 61, 054003 (2000); 70, 035209 (2004).

32. G. Caia, V. Pascalutsa, J.A. Tjon, L.E. Wright, Phys. Rev. C 70, 032201(R) (2004); G. Caia, L.E. Wright, V. Pascalutsa, Phys. Rev. C 72, 035203 (2005).

33. S. Weinberg, Physica A 96, 327 (1979).

34. J. Gasser, H. Leutwyler, Ann. Phys. (N.Y.) 158, 142 (1984).
35. E. Jenkins, A.V. Manohar, Phys. Lett. B 255, 558 (1991); 259, 353 (1991).

36. T. Hemmert, B.R. Holstein, J. Kambor, Phys. Lett. B 395 89 (1997); G. Gellas et al., Phys. Rev. D 60, 054022 (1999).

37. H.-B. Tang, P. Ellis, Phys. Lett. B 387, 9 (1996).

38. N. Fettes, U.G. Meissner, Nucl. Phys. A 679, 629 (2001).

39. V. Pascalutsa, D.R. Phillips, Phys. Rev. C 67, 055202 (2003); 68, 055205 (2003).

40. C. Hacker, N. Wies, J. Gegelia, S. Scherer, Phys. Rev. C 72, 055203 (2005).

41. V. Pascalutsa, M. Vanderhaeghen, Phys. Rev. Lett. 95 232001 (2005).

42. V. Pascalutsa, M. Vanderhaeghen, arXiv:hep-ph/0512244.

43. V. Pascalutsa, M. Vanderhaeghen, Phys. Rev. Lett. 94, 102003 (2005).

44. V. Pascalutsa, M. Vanderhaeghen, arXiv:hep-ph/0511261.

45. J. Gasser, M.E. Sainio, A. Svarc, Nucl. Phys. B 307, 779 (1988).

46. W. Rarita, J.S. Schwinger, Phys. Rev. 60, 61 (1941).

47. V. Pascalutsa, Phys. Rev. D 58, 096002 (1998).

48. V. Pascalutsa, R.G.E. Timmermans, Phys. Rev. C 60 , 042201(R) (1999).

49. V. Pascalutsa, Phys. Lett. B 503, 85 (2001).

50. S. Deser, V. Pascalutsa, A. Waldron, Phys. Rev. D 62 , 105031 (2000).

51. J. Gegelia, G. Japaridze, Phys. Rev. D 60, 114038 (1999); J. Gegelia, G. Japaridze, X.Q. Wang, J. Phys. G 29, 2303 (2003).

52. C.W. Bernard et al., Phys. Rev. D 64, 054506 (2001).

53. R.A. Arndt, W.J. Briscoe, I.I. Strakovsky, R.L. Workman, Phys. Rev. C 66, 055213 (2002).

54. C. Kunz et al., Phys. Lett. B 564, 21 (2003).

55. K.M. Watson, Phys. Rev. 95, 228 (1954).

56. T. Pospischil et al., Phys. Rev. Lett. 86, 2959 (2001).

57. D. Elsner et al., arXiv:nucl-ex/0507014.

58. C. Alexandrou et al., Phys. Rev. Lett. 94, 021601 (2005).

59. B.M.K. Nefkens et al., Phys. Rev. D 18, 3911 (1978).

60. A. Bosshard et al., Phys. Rev. D 44, 1962 (1991).

61. D. Drechsel, M. Vanderhaeghen, M.M. Giannini, E. Santopinto, Phys. Lett. B 484, 236 (2000).

62. M. Kotulla et al., Phys. Rev. Lett. 89, 272001 (2002).

63. D. Drechsel, M. Vanderhaeghen, Phys. Rev. C 64, 065202 (2001).

64. W.T. Chiang, M. Vanderhaeghen, S.N. Yang, D. Drechsel, Phys. Rev. C 71, 015204 (2005).

65. R. Beck, B. Nefkens, spokespersons Crystal Ball at MAMI experiment.

66. Particle Data Group (K. Hagiwara et al.), Phys. Rev. D 66, 010001 (2002).

67. D.B. Leinweber, T. Draper, R.M. Woloshyn, Phys. Rev. D 46, 3067 (1992); I.C. Cloet, D.B. Leinweber, A.W. Thomas, Phys. Lett. B 563, 157 (2003).

68. F.X. Lee, R. Kelly, L. Zhou, W. Wilcox, arXiv:heplat/0410037.

69. C. Alexandrou et al., arXiv:hep-lat/0509140. 